\title{
TRANSVERSE FIELD IMPLIES NORMAL MICROBUNDLE
}

\author{
H. PUTZ
}

The main result of this paper is the following theorem.

THEOREM. Let $M^{n}$ denote a nonbounded combinatorial manifold rectilinearly imbedded in some Euclidean space. If $M^{n}$ possesses a transverse field, then $M^{n}$ has a normal microbundle.

We assume that the reader is familiar with the theory of piecewise linear microbundles, which was developed by J. Milnor (see [5] or [6]), as well as familiarity with the theory of $C^{1}$-complexes, developed by J. H. C. Whitehead, (see [7] or [10]).

0 . Convention. Throughout this paper $M^{n}$ will denote a nonbounded combinatorial manifold rectilinearly imbedded in some Euclidean space.

1. Definition. Let $s$ denote a simplex (open) of $M$. By $P(s)$ we will denote the subset (possibly empty) in $G_{n, p}$ (the Grassmannian manifold of all $p$-planes through the origin in $R^{n+p}$ ) consisting of all $p$-planes $P$, such that, if $H$ is the orthogonal $n$-plane to $P$ in $R^{n+p}$, then the orthogonal projection $q: R^{n+p} \rightarrow H$, restricted to $\operatorname{St}(s, M)^{-}$ (the closed star of $s$ in $M$ ) is a homeomorphism carrying $\operatorname{St}(s, M)$ onto an open set in $H$. If the $p$-plane $P$ belongs to $P(s)$, then $P$ is said to be transversal to $M$ at $m$, where $m$ is any point of $s$. A continuous map $g: M \rightarrow G_{n, p}$ is a transverse field, if the set $g(s)$ is contained in the set $P(s)$, for every simplex $s$ of $M$.

2. Remark. If the simplex $t$ is a face of the simplex $s$, then since $\operatorname{St}(s, M)^{-}$is contained in $\operatorname{St}(t, M)^{-}$, we have the fact that the set $P(t)$ is contained in the set $P(s)$.

3. Lemma. Let $g: M^{n} \rightarrow G_{n, p}$ be a transverse field. Without loss of generality we may assume that the map, $g$, is piecewise smooth.

Proof. Suppose we have a continous transverse field, $h: M^{n} \rightarrow G_{n, p}$. By Remark 2 we see that $h(\bar{s})$ is contained in $P(s)$. The reader can verify that the sets $P(s)$ are open in $G_{n, p}$ (or see Proposition 3.7 of [9]). Thus, since $h(\xi)$ is compact, we see that there exists a positive continuous function $\delta$ mapping $M$ to the positive reals, such that, if

Received by the editors September 16, 1968 and, in revised form, December 13, 1968 and April 11, 1969. 
$g: M \rightarrow G_{n, p}$, and $\|g(x)-h(x)\|<\delta(x)$ (where \|\| denotes a metric on $\left.G_{n, p}\right)$, then $g(s)$ is contained in $P(s)$. Now let $V$ denote the second regular neighborhood of $M$ in $R^{n+p}$, and let $r: V \rightarrow M$ denote the retraction of this open set onto $M$. Thus we have a continuous map $h r: V \rightarrow G_{n, p}$ defined on the smooth manifold $V$. Let $g: V \rightarrow G_{n, p}$ be a , smooth map such that its restriction to $M$ is pointwise within $\delta(x)$ of the map $h$. (Such a map exists by the proof of Theorem 4.2 of [4].) If we let the restriction map also be denoted by $g$, we have the proof of the lemma.

4. Lemma. Given a transverse field $g: M \rightarrow G_{n, p}$. Let $N$ denote the subset of $M \times R^{n+p}$ consisting of pairs $(x, v)$ such that the vector $v$ belongs to the p-plane $g(x)$. Then $N$ is a vector bundle with base $M$, which we shall call a normal bundle of the imbedding of $M$ in $R^{n+p}$.

Proof. Consider the $p$-dimensional vector bundle $G$ consisting of pairs $(P, v)$ in the space $G_{n, p} \times R^{n+p}$ such that the vector $v$ belongs to the $p$-plane $P$. The set $N$ then, is just the bundle induced by the map $g$.

5. Remark. Let $g: M \rightarrow G_{n, p}$ be a piecewise smooth transverse field. (This is no loss in generality by Lemma 3.) The map $g$ induces a map $f: N \rightarrow R^{n+p}$ defined by $f(x, v)=x+v$. Let $m$ be a point of $M$, then since a vector bundle over a contractible set is trivial, let $h: \operatorname{St}(m, M)^{-}$ $\times R^{p} \rightarrow N$ denote a trivialization of $N$ over $\operatorname{St}(m, M)^{-}$. The map $h$ may be chosen such that the map $k: \operatorname{St}(m, M)-\times R^{p} \rightarrow R^{n+p}$ induced by $f$ is piecewise smooth. (We give $\operatorname{St}(m, M)^{-} \times R^{p}$ a simplicial structure.) Let $e_{1}, \cdots, e_{p}$ denote an orthonormal basis of $R^{p}$, and set $h\left(x, e_{i}\right)=\left(x, v_{i}(x)\right)$, a point of $N$. Then for a point $w$ in $R^{p}$ such that $w=\sum c_{i} e_{i}$, we have that $k(x, w)=x+\sum c_{i} v_{i}(x)$. Finally we define a new map $k^{\prime}: \operatorname{St}(m, M)^{-} \times R^{p} \rightarrow R^{n+p}$ by $k^{\prime}(x, w)=x+\sum c_{i} v_{i}(m)$.

6. Lemma. The map $k^{\prime}: \operatorname{St}(m, M)-\times R^{p} \rightarrow R^{n+p}$ defined in the previous remark is a piecewise smooth imbedding.

Proof. Let $X=\operatorname{St}(m, M)-\times R^{p}$ and $b=(x, w)$. We must prove that: (1) $d k_{b}^{\prime}: \operatorname{St}(b, X)^{-} \rightarrow R^{n+p}$ is one-to-one for each point $b$ of $X,(2) k^{\prime}$ is a homeomorphism.

Proof of (1). Recall that $d k_{b}^{\prime}(z)=D k^{\prime}(b) \cdot(z-b)$ (where $D k^{\prime}(b)$ is the Jacobian of the map $k^{\prime}$ with respect to a simplex containing $b$. Though the Jacobian depends upon the simplex chosen, the map $d k_{b}^{\prime}$ does not). To prove that the map $d k_{b}^{\prime}$ is one-to-one, it is obviously sufficient to prove that the Jacobian $D k^{\prime}(b)$ is nonsingular. Now the $n+p \times n+p$ matrix $D k^{\prime}(b)=\left(C ; v_{1}(m) ; \cdots ; v_{p}(m)\right)$, where 
the $n$ columns of the $n+p \times n$ matrix $C$ span the $n$-plane determined by an $n$-simplex in $\operatorname{St}(m, M)^{-}$containing the point $x$. Since the vectors $v_{i}(m), i=1, \cdots, p$ span the $p$-plane $g(m)$ and are not in the $n$-plane determined by any simplex of $\operatorname{St}(m, M)^{-}$, the Jacobian $D k^{\prime}(b)$ is nonsingular.

Proof of (2). It is easily seen that it suffices to prove that the map $k^{\prime}$ is one-to-one. If $k^{\prime}$ is not one-to-one, then there exist pairs $(x, w)$ and $(y, u)$, where $w=\sum c_{i} e_{i}$ and $u=\sum d_{i} e_{i}$, such that $x+\sum c_{i} v_{i}(m)$ $=y+\sum d_{i} v_{i}(m)$. This implies that the vector $x-y$ belongs to the $p$-plane $g(m)$, and hence that the projection of $\operatorname{St}(m, M)^{-}$to the orthogonal $n$-plane to $g(m)$ is not one-to-one. This contradicts the choice of $g(m)$.

7. Lemma. Given a point $m$ of $M$, there exists a finite subcomplex $Y$ (depending on the point $m$ ) of some subdivision of $\operatorname{St}(m, M)^{-} \times R^{p}$, which contains a neighborhood of $(m, 0)$ on which the map $k$ (defined in Remark 5) is a piecewise smooth imbedding.

Proof. Let $Z$ be a finite subcomplex of $\operatorname{St}(m, M)^{-} \times R^{p}$ consisting of pairs $(x, w)$ such that $\|w\| \leqq 1$. By Theorem 8.8 of $[7]$, and the previous lemma, there is a $\delta>0$, such that any $\delta$-approximation to the map $k^{\prime}$ is a piecewise smooth imbedding, for any subcomplex $Y$ contained in an arbitrary subdivision of $Z$. To insure that the map $k$ is the desired $\delta$-approximation, we choose $Y$ as follows. First, for $w=\sum c_{i} e_{i}$ in $R^{p}$, let $w(x)=\sum c_{i} v_{i}(x)$ in $R^{n+p}$. Now we have that $\left\|k(x, w)-k^{\prime}(x, w)\right\|=\|w(x)-w(m)\|$. Thus on $Z$, since $\|w\| \leqq 1$, we may choose the point $x$ sufficiently close to $m$, to insure that $\|w(x)-w(m)\|<\delta$. We next wish to choose $b=(x, w)$ sufficiently close to $(m, 0)$ so that $\left\|d k_{b}(z)-d k_{b}^{\prime}(z)\right\|<\delta\|z-b\|$, or equivalently so that $\left\|D k(x, w)-D k^{\prime}(x, w)\right\|<c \delta$. (The norm of a matrix is the absolute value of its maximum entry; $c=(n+p)^{3 / 2}$.)

Now $D k(x, w)=\left(C+c_{1} B_{1}(x)+\cdots+c_{p} B_{p}(x) ; v_{1}(x) ; \cdots ; v_{p}(x)\right)$ where the $n$ columns of the $n+p \times n$ matrix $C$ span the $n$-plane determined by an $n$-simplex in $\operatorname{St}(m, M)^{-}$-containing $x$, and the entries of the $n+p \times n$ matrices $B_{i}(x)$ are bounded. Since $D k^{\prime}(x, w)$ $=\left(C ; v_{1}(m) ; \cdots ; v_{p}(m)\right)$ we have that $\left\|D k(x, w)-D k^{\prime}(x, w)\right\|$ $=\left\|\left(c_{1} B_{1}(x)+\cdots+c_{p} B_{p}(x) ; v_{1}(x)-v_{1}(m) ; \cdots ; v_{p}(x)-v_{p}(m)\right)\right\|$.

Thus choose $\|w\|$ small enough, and $x$ sufficiently close to $m$ to insure the desired inequality. (Of course the Jacobians chosen depend upon the $n+p$ simplex chosen to contain the point $(x, w)$, but there are only a finite number of such simplices.) Let $U$ be the neighborhood of $(m, 0)$ so determined to insure the above inequalities. Subdivide the complex $Z$ sufficiently fine to obtain the desired complex $Y$ contained in $U$. 
8. Corollary. There is a neighborhood of the zero section of the normal bundle $N$ of $M$ on which the map $f: N \rightarrow R^{n+p}$ induced by the piecewise smooth transverse field $g: M \rightarrow G_{n, p}$ is a homeomorphism which maps this neighborhood onto an open subset of $R^{n+p}$.

Proof. By Lemma 5.7 of [7], it is sufficient to prove that each point $(m, 0)$ in the zero section of $N$ has a neighborhood which $f$ maps homeomorphically onto an open set of $R^{n+p}$. But this holds by Lemma 7 , the desired neighborhood being the interior of the complex $Y$. The image is open, for if $m$ belongs to the simplex $s$, the fact that $P(s)$ is nonempty implies that $\operatorname{St}(m, M)^{-} \times R^{p}$, which equals $\operatorname{St}(s, M)^{-} \times R^{p}$. is homeomorphic with a subset of Euclidean $n+p$ space. Thus Brouwer's theorem on the invariance of domain [3] implies that the image is open.

Let $V$ denote the neighborhood of the zero section of the normal bundle $N$ which by the previous corollary, $f$ maps homeomorphically onto an open set $f(V)$ in $R^{n+p}$. We shall give $f(V)$ a microbundle structure so that it is a normal microbundle of $M$. In order to do this we need the result that every vector bundle has a microbundle structure (see [4] or [8]). In fact, we use not only the result, but also the proof of that result as presented in [8].

9. Theorem. Let $M^{n}$ denote a nonbounded combinatorial manifold rectilinearly imbedded in some Euclidean space. If $M^{n}$ possesses a transverse field, then $M^{n}$ has a normal microbundle.

Proof. Let $e: K \rightarrow N$ be the triangulation of the normal bundle $N$ given in [8]. The simplicial complex $K$ is thus a microbundle over $M$. Let $L=e^{-1}(V)$. According to $[1$, p. 143] we may choose a rectilinear triangulation of $L$ such that the incluslon map of $L$ into $K$ is piecewise linear. The map $f e: L \rightarrow R^{n+p}$ is a piecewise smooth imbedding. This is due to the fact that in constructing the triangulation $(e, K)$ of the normal bundle $N$, we may, as in [8], start with the piecewise smooth imbeddings $h: \operatorname{St}(m, M) \times R^{p} \rightarrow N$, where $m$ is a vertex of $M$. The maps $h$ are then changed slightly to maps $h^{\prime}$ which we choose sufficiently close to the maps $h$ to insure that the map $f e$, which locally is the map $f h^{\prime}$, is a piecewise smooth imbedding. (We leave the details to the reader, noting however, that for a detailed proof, Lemma 7 is essential.) Now let $d: L \rightarrow R^{n+p}$ denote a piecewise linear approximation to the map $f e$, sufficiently close to insure that it is a piecewise smooth imbedding, and that $d(L)=f e(L)=f(V)$, an open set in $R^{n+p}$. To show that $d(L)$ is a microbundle with base $M$, it is sufficient to show that $L$ is a microbundle with base $M$. But any open neighborhood of the zero section of a microbundle is a microbundle over the 
base. (In the definition of a microbundle we may replace $R^{q}$ by any open ball about the origin, since the two are piecewise linearly homeomorphic.) Thus $d(L)$ is a normal microbundle for $M$, the piecewise linear map $j: d(L) \rightarrow M$ is that induced by the piecewise linear projection $K \rightarrow M$.

We now prove a corollary to this theorem. Since this corollary is independent of the global codimension of the imbedding of $M$ it differs from results of A. Haefliger and C. T. C. Wall [2].

10. Definition. The imbedding of $M^{n}$ in $R^{n+p}$ is locally normal if for each vertex $m$ of $M$ the set $P(m)$ is nonempty. Hence by Remark 2 the set $P(s)$ is nonempty for each simplex $s$.

11. Definition. The imbedding of $M^{n}$ in $R^{n+p}$ is of local codimension $k$ if for each vertex $m$ of $M$ there is an $n+k$ plane $J$ (depending on $m$ ) such that $\operatorname{St}(m, M)$ is contained in some translation of $J$. (This suggestive terminology of local codimension is due to H. S. Davis.)

12. Corollary to Theorem 9. Let $M^{n}$ denote a nonbounded combinatorial manifold rectilinearly imbedded in some Euclidean space. If the imbedding is locally normal and of local codimension 1 , then $M^{n}$ possesses a normal microbundle.

Proof. The principal result of [9, Theorem 1.8], states that the hypothesis implies that $M^{n}$ has a transverse field. Hence by Theorem $9, M^{n}$ possesses a normal microbundle.

\section{REFERENCES}

1. P. Alexandroff and H. Hopf, Topologie, Springer, Berlin, 1935.

2. A. Haefliger and C. T. C. Wall, Piecewise linear bundles in the stable range, Topology 4 (1965), 209-214.

3. W. Hurewicz and H. Wallman, Dimension theory, Princeton Univ. Press, Princeton, N. J., 1941.

4. R. Lashof and M. Rothenberg, Microbundles and smoothing, Topology 4 (1965), 357-388.

5. J. Milnor, Microbundles and differentiable structures, (mimeographed notes), Princeton Univ., Princeton, N. J., 1961.

6. —, Microbundles, Toplogy 3 (1964), 53-80.

7. J. R. Munkres, Elementary differentiable topology, Ann. of Math. Studies, No. 54, Princeton Univ. Press, Princeton, N. J., 1963.

8. H. Putz, Triangulation of fibre bundles, Canad. J. Math. 19 (1967), 499-513.

9. - Construction of transverse fields, Canad. J. Math. (to appear).

10. J. H. C. Whitehead, On $C^{1}$-complexes, Ann. of Math. 41 (1940), 809-824.

TEMPLE UNiversity 